\title{
Numerical and Experimental Study of Mechanical Oscillation Energy
}

\author{
Andrej LIPEJ*, Matej ŠTEFANIČ, Simon MUHIČ, Primož KUNAVER
}

\begin{abstract}
The paper presents an interesting opportunity to use a wide spread available energy source that has not yet been fully utilized. A new generator solution will be studied to produce electricity from oscillation energy. The solution will be useful for various purposes, especially for shipping, as well as freight road and rail transport. With this solution, it will be possible to reduce the usage of some amount of fossil fuels globally. Research includes two different theoretical assumptions. First approach involves the conversion of energy using Torus Type Generator (TTG) and second using Eccentric Type Generator (ETG). In TTG case, a new type of generator in which a magnetic cylinder moves freely in a hollow torus with electrical winding has been analysed. In the case of an ETG generator, a physical pendulum with a vertical axis of rotation has been analysed. Experimental and numerical methods were used for research. For the numerical analysis of 3D body motion the ANSYS Rigid Body Dynamics software was used.
\end{abstract}

Keywords: experimental study; numerical analysis; oscillation energy; rigid body mechanics

\section{INTRODUCTION}

The research area covered by the presented paper is quite rarely discussed in scientific papers. Most research is related to addressing wave energy conversion $[1,2]$. Some research focuses on more technical and economic issues [3] or on optimizing the energy conversion process [4].

The basic theory is fairly well discussed in the literature [5-7], but in complex cases the use of modern numerical methods is absolutely necessary because analytical solutions in most concrete cases cannot be obtained.

The initial concept of operation of the TTG generators is closest to linear generators [8-10], of which several variants have been developed in the past. As a rule, existing versions of linear generators transfer external excitation in proportion to the amplitude and frequency of excitation into the internal amplitude and frequency of motion of the magnetic core after the coil is wound. This, however, has limited potential of applications, which is certainly one of the main reasons why the use of fluctuations in transport, using current technological solutions, has not been economically interesting to exploit so far.

When accelerating, braking or oscillating, a magnetic cylinder moves inside the torus and induces electrical voltage. This type of generator will have a high efficiency and will be particularly suitable for larger systems.

Generator ETG represents a new version of the multiphase generator, which is based on an eccentric mass that rotates around the axis. The concept of a solution is similar to the clock with automatic winding, and for this purpose working solutions already exist which, however, use mechanical transmissions.

Standard generators are not used for the purpose described. The paper presents basic concepts of both solutions with some preliminary numerical end experimental investigation of mechanical and electrical characteristics.

In our case, the great benefit in terms of innovation and efficiency of the two presented concepts is that they allow a much longer path of the magnetic core. Namely, with the help of horizontal positioning, a small displacement from equilibrium position with low acceleration, deceleration or inclination of the device, enables our solution to induce multiple rotations of the magnetic core along the torus or circular through the coil winding.

To achieve this, it will be necessary to ensure minimal slip or rolling friction of the magnetic core, which will be one of the technological challenges of the project. The vibration energy of ships due to waves and the acceleration, deceleration and oscillation of freight vehicles, are given which, in the current state of technology is in fact only a disruptive factor, without any benefit.

Utilizing this energy would provide a virtually free and environmentally acceptable additional source of electricity that could cover part of the transporter's own energy needs. We consider the first most interesting segment of use to be freight shipping, the use in road freight transport is also interesting, and the prospects of use are in passenger ship transport, passenger cars and rail transport.

Numerical analysis of free motion of rigid bodies is restrained by different boundary conditions like loads and supports. Special role in the analysis have links of two bodies. These joints are idealizations of the contact between the bodies. The primary unknowns of a rigid dynamics solution are the translation and rotation of each body and the motion in the joints themselves.

The output quantities of rigid body dynamics are the forces that develop in the joints and flow through the rigid bodies, as opposed to a structural analysis where the output quantities are strains or stresses. Additional outputs are also torque, velocity and acceleration of rigid bodies.

The developed generator solutions will generate electricity which will be used for own use of the vehicle. This means that the total fuel consumption (the consumption is very different depending on the type of vessel) can be reduced. In road freight, the fuel consumption for the alternator drive is approx. $2 \%$, or 0,5 litres of fuel per hour of driving. Globally, in 2015, 298 million tons of fuel oil was used annually for shipping, generating 932 million tons of $\mathrm{CO}_{2}$ emissions into the atmosphere. Any reduction in greenhouse gas emissions will be very welcome in the near future.

\section{DRIVEN OSCILLATION OF GENERATORS}

Both types of generators ETG and TTG are considered essentially as fairly simple pendulums. Each pendulum has 
its natural frequency $\omega_{\mathrm{g}}$ according to its properties, if the weight is moved away from the equilibrium position.

There is always some resistance to oscillation that inhibits oscillation, and in such a case there is talk of damped oscillation. In the case of both of the above generators, there are several causes of damping. Friction, air resistance and electromagnetic forces must be taken into account when operating the generator. All influences can be combined into a single damping coefficient $\beta$.

One of the most important tasks is how to minimize the influence of all resistant forces, which influence the motion of magnet inside the torus or motion of the ETG weight.

With damped oscillations, the energy decreases exponentially with time

$$
W=W_{\mathrm{o}} \mathrm{e}^{(-2 \beta t)}
$$

It makes sense to talk about the oscillation energy if the natural oscillation time is much shorter than the time $1 / \beta$, in which the oscillation energy changes significantly. In this case, the pendulum oscillates several times before the oscillation is significantly dumped. Thus, the average oscillation energy over one oscillation can be defined.

In that case, the behaviour of such a pendulum which is in a coordinate system that also oscillates with its own $\omega_{\mathrm{cs}}$ frequency is considered. Supposedly we are dealing with forced oscillation, where in certain cases both frequencies can be very similar, the appearance of resonance can be expected.

In the case of forced oscillation, the maximum amplitude of oscillation also changes. The pendulum whose natural frequency is closest to the frequency of the driven oscillation, oscillates with the largest amplitude.

The change in amplitude with respect to different frequencies of forced oscillation and different coefficients of resistance can be represented by a resonance curve. Since the conversion of mechanical oscillation energy into electrical energy is interesting, it is also important to know the ratio of the average power supplied to the pendulum $\left(P_{\text {in }}\right)$ and the actual power $(P)$ of the excited oscillation.

This relationship can be represented by the following equation:

$$
\frac{P}{P_{\text {in }}}=\frac{(x)^{2}}{\left[\left(1-(x)^{2}\right)^{2}+\left(\frac{2 \beta}{\omega_{\mathrm{g}}}\right)^{2}(x)^{2}\right]}
$$

where $x$ is defined as a ratio of natural and forced circular frequency

$$
x=\frac{\omega_{\mathrm{cs}}}{\omega_{\mathrm{g}}}
$$

The curve described by the above equation has a maximum at circular frequency of the coordinate system, which is equal to circular frequency of pendulum $\left(\omega_{\mathrm{g}}\right)$, regardless of the size of the damping coefficient.

Thus, the output power of the oscillating generator can be expected to be greater than only the available potential energy taken into account. In this case, of course, the energy of oscillation is not taken into account.

In certain cases, such as the treatment of the abovementioned types of generators, the body moves on the surface of another stationary body under the influence of weight. In such a case, its motion is limited to a specific surface in $3 \mathrm{D}$ space.

If the body is moving e.g. in a tube, along a torus, etc., its motion is limited to a curve given by $r(s)$, where $s$ is the path (distance) along the curve. The particle, which is restricted in its motion by the bonds, falls due to the limitations of the (additional) force, which can be called the bonding forces.

It is therefore appropriate to divide the forces into active forces and bond forces $\left(F_{\mathrm{b} i}\right)$ when considering the motion in detail

$\overline{F_{i}}=\overline{F_{\mathrm{a} i}}+\overline{F_{\mathrm{b} i}}$

where the active forces $F_{\mathrm{a} i}$ directly affect the motion in the allowed coordinate space.

The general motion of a different number of particles in 3D space can be described using Newton's basic equations for a particle system

$m_{i} \ddot{r}_{i}=\overline{F_{i}}=\overline{F_{\mathrm{a} i}}+\overline{F_{\mathrm{b} i}}$

In the above equation $m_{i}$ represents the mass of individual particle and $r_{i}$ the radius vector of the position in coordinate system.

Due to the complexity of general motion in 3D space, numerical simulations were used for analysis of the general motion of a rigid body to solve this problem.

\section{TORUS TYPE GENERATOR}

The basic assumption in the movement of a magnet in the torus (Fig. 1) is the periodic variation of the torus slope, which may be very similar to a sine wave, or to have an arbitrary time course. The frequencies and amplitudes of oscillations can change.
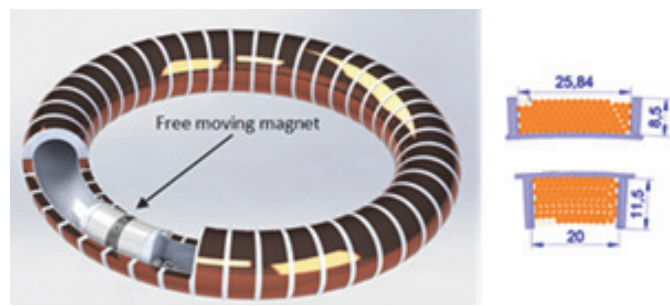

Figure 1 Basic concept of Torus Type Generator with coil winding layout

For basic kinematics, where no external forces are taken into account, the amplitudes are not so important because the magnet starts to move as soon as the oscillation begins.

At very low frequencies, the magnet moves out of the rising part of the torus and swings at the lowest part. The process is repeated at some frequency interval. At a certain frequency, it can be seen that the magnet begins to rotate in the torus.

Speed of rotation varies at a certain interval of frequencies and amplitudes. If the frequency is very high, 
the magnet may only move on a limited part of the torus, or it may be almost stationary.

Due to the multitude of different situations, a simple scaled-down model which was tested in the lab and roughly measured the impact of all possible variables was made.

The required oscillation frequency and amplitude to obtain the most optimal magnet motion were determined.

In case of movement of the magnet in the torus, all forces that inhibit the movement must be considered. These forces will influence the determination of optimal frequencies and amplitudes.

Fig. 2 shows the basic geometry used for numerical simulation with Ansys Rigid Body Dynamics software [11]. With different input parameters regarding the oscillation amplitudes and frequencies and also taking into account the losses of energy during the motion, a lot of calculations was made.

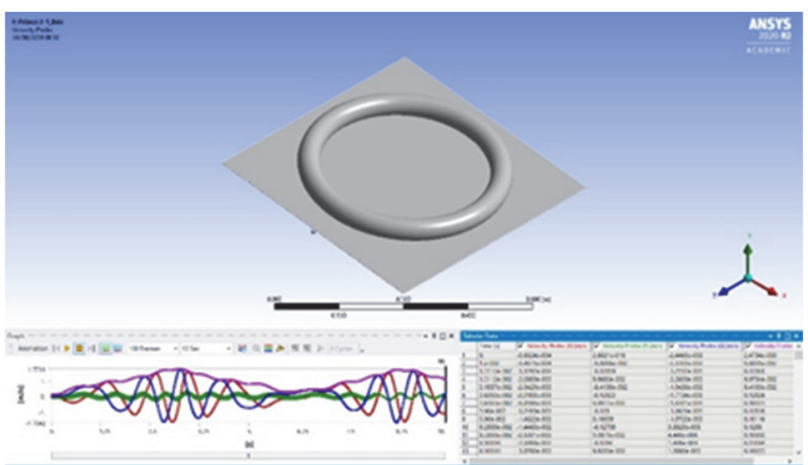

Figure 2 Numerical prediction of the motion inside the torus - geometry

The losses were simulated using the friction coefficient between the magnet and the torus walls. The true value of the friction coefficient was obtained by comparing the experimental and numerical results under the same oscillation conditions.

Analysis of results shows large differences between individual modes of movement. If only potential energy is considered as input energy due to the different heights of the position of the magnet, it can be concluded that the energy obtained is even greater than the initial potential energy. This would certainly not be possible if the oscillation energy that is at our disposal under the basic assumption without major limitations has not been neglected.

The limitations are mainly in the ratio between the mass of the moving magnet and the mass of the oscillating plant where the generator is installed. It is supposed that the mass of the magnet is negligible or for several orders of magnitude smaller than the mass of the whole plant.

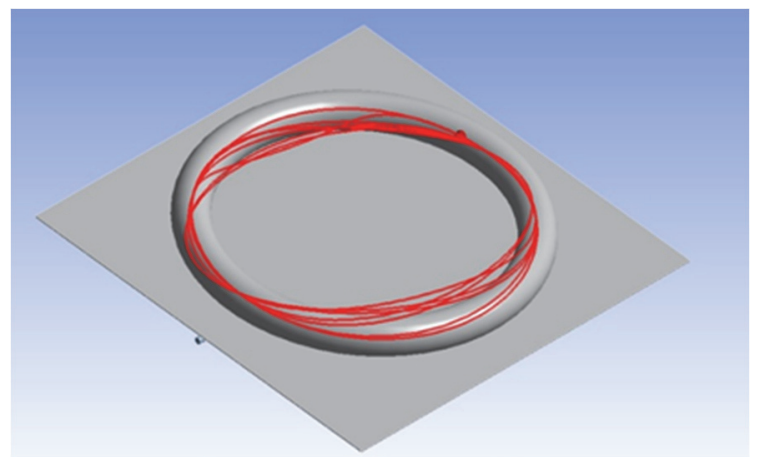

Figure 3 Numerical prediction of the motion inside the torus - magnet motion
This was proved by numerical analyses (Fig. 2) and by laboratory measurements (Fig. 6). Fig. 3 shows the trajectories of motion within the torus when the amplitude and frequency are close to the optimal solution and a fairly uniform magnet rotation is obtained.

Because the torus changes height, circular trajectories do not mean that the rotation is at a constant rotational speed, which can be seen in the results of numerical analyses (Fig. 4 and Fig. 5).

In numerical analyses, the time course of the magnet velocity was mainly analysed, while in laboratory measurements, the maximum power was measured by measuring voltage and electric current.

Fig. 4 presents the results for the first case. It can be seen that the time distribution of velocity is very uneven and the maximal velocity is less than $0,7 \mathrm{~m} / \mathrm{s}$.

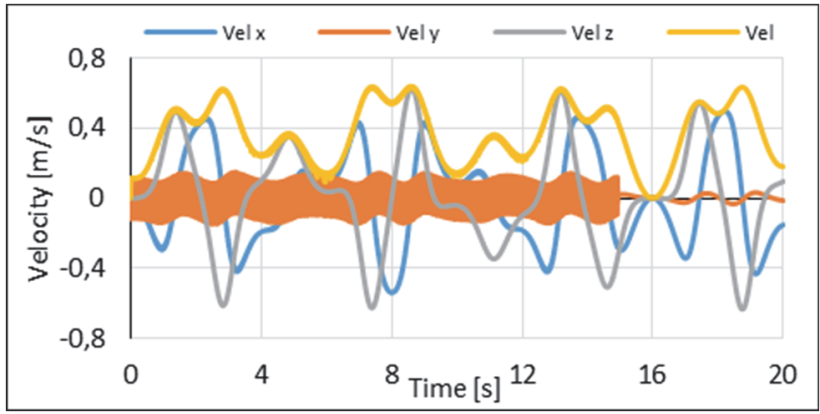

Figure 4 Numerical prediction of the motion inside the torus - case one - velocity of the magnet

The maximal velocity in the second case is much higher, more than $100 \%$ (Fig. 5).

Numerical analysis was performed for a larger number of frequencies and amplitudes, where the values of these varied by more than $100 \%$. Change in the frequency and amplitude of the oscillation can increase the maximum speed of the magnet by about $200 \%$.

It should be emphasized that, of course, the maximum power is only one part of the result, which is necessary for the efficient operation of the generator.

It is also necessary to analyse the time course of all movements, according to different frequencies and amplitudes, and to estimate the production of electricity in a longer time interval.

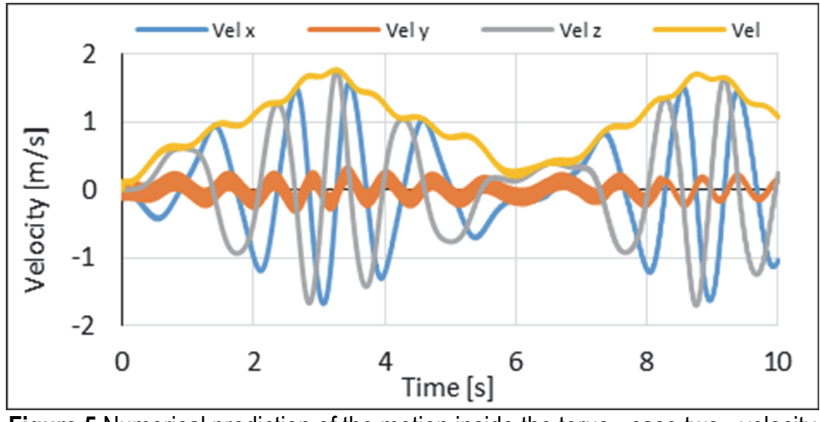

Figure 5 Numerical prediction of the motion inside the torus - case two - velocity of the magnet

Only this information will actually be an indicator of the usefulness of the torus generation. In the first part of the research, the focus was mainly on determining the conditions for achieving maximum power. 
The power output from mechanical oscillation generators may be increased by optimal controlling of the oscillation.

Numerical results were confirmed by laboratory measurements. The maximum measured power of the generator with identical dimensions as in numerical analyses differed by less than $10 \%$. Given the fact that only mechanical parameters were considered in the numerical analyses, the result is quite good.

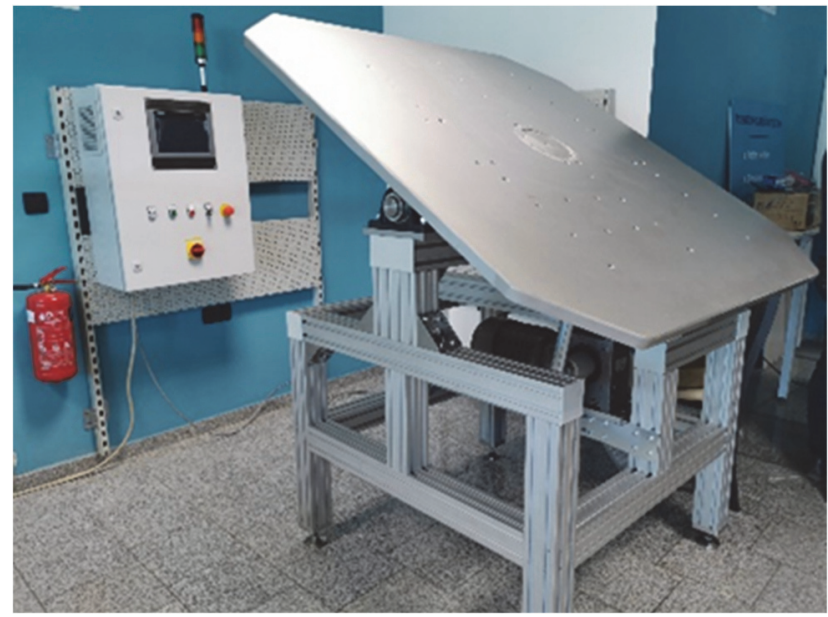

Figure 6 Test rig for oscillation simulation layout

The model size of the TTG generator was the same for numerical and experimental analysis.

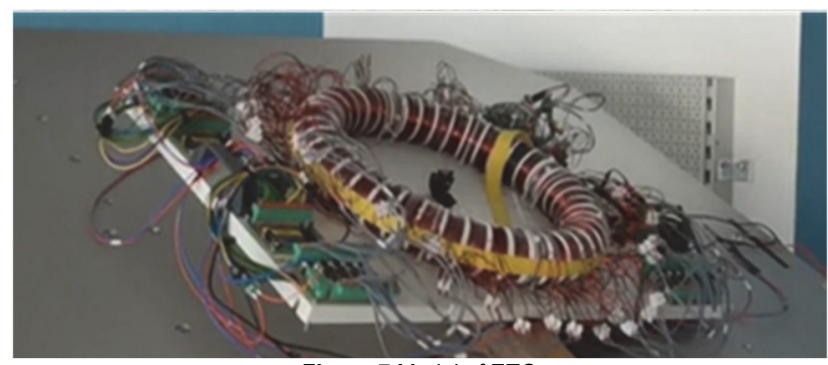

Figure 7 Model of ETG

The diameter of the torus was $0,5 \mathrm{~m}$, the mass of the magnet was $1 \mathrm{~kg}$, and the diameter of the torus cross section was $0,047 \mathrm{~m}$ (Fig. 7).

\section{GENERATOR TYPE ECCENTRIC}

The ETG can be imagined as a relatively simple physical pendulum with a vertical axis of rotation. If such a pendulum is moved away from its equilibrium position, it oscillates with some natural frequency, which depends on the physical properties of the pendulum.

Such oscillation is subject to the conservation of full energy, so that in the case where losses are not taken into account, the full energy of oscillation is preserved. In our case, the total oscillation energy is equal to the maximum potential energy, which is conditioned by the maximum height difference of the weight (Fig. 8).

In the case of an ETG, full energy consists of the sum of potential and kinetic energy, depending on the position of the weights of the ETG.

To obtain the information about the motion the movement of eccentric weights using the ANSYS Rigid
Body Motion software package was investigated numerically.

Initially, the 3D geometry of all moving parts was defined. Forced oscillation of the pendulum was initially given by the classical cosine function, but later it was found out that much more realistic results, if the frequency of oscillation changes in time, were obtained.

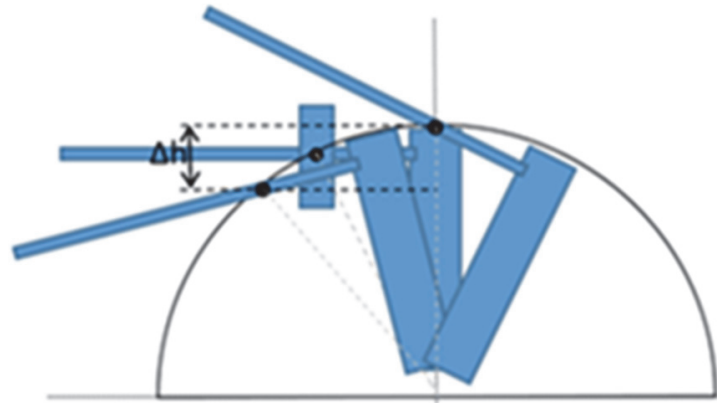

Figure 8 Definition of maximum height difference - model of ETG

The time course of the excited oscillation is seen in Fig. 9 where the red curve of the forced oscillation is shown.

The maximum amplitude of the oscillation and a friction coefficient on the rotating shaft were also defined.

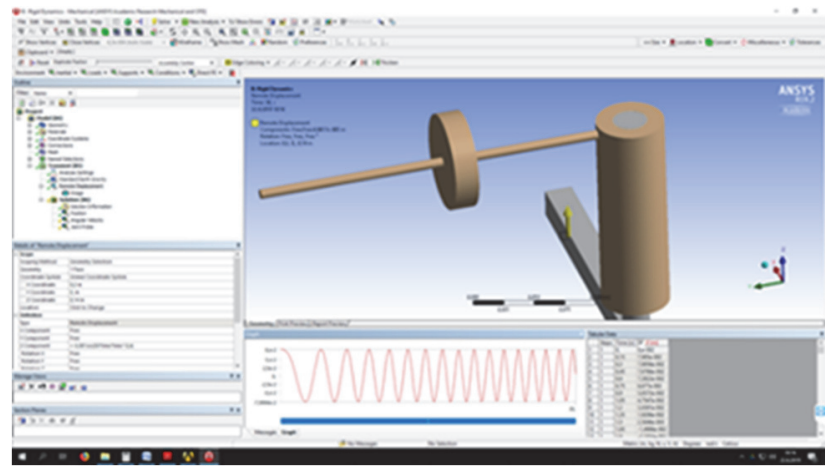

Figure 9 Numerical simulation of ETG

The trajectory of the centre of gravity of the moving weight depends very much on the oscillation parameters. In some cases, an almost uniform circulation (Fig. 10) was obtained where energy conversion is most efficient.

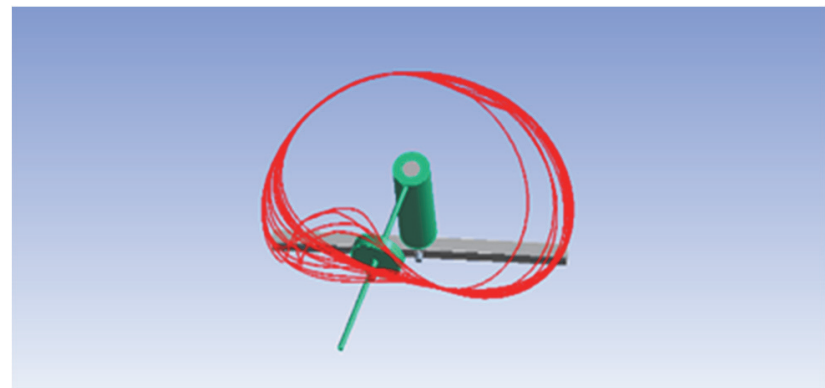

Figure 10 Numerical prediction of the weight motion

As a result of the numerical simulation of the eccentric motion based on the utilization of potential energy, we obtained the time distribution of the coordinate of the centre of gravity, angular velocity (Fig. 11) and torque on the rotating shaft (Fig. 12).

Under most initial and boundary conditions, the movement of the centre of gravity was very chaotic. The 
weight could swing a little, move in one direction or the other, or stop rotating around the axis and just swing up and down. In such a case no induced voltage is obtained and this means that such conditions should be avoided.

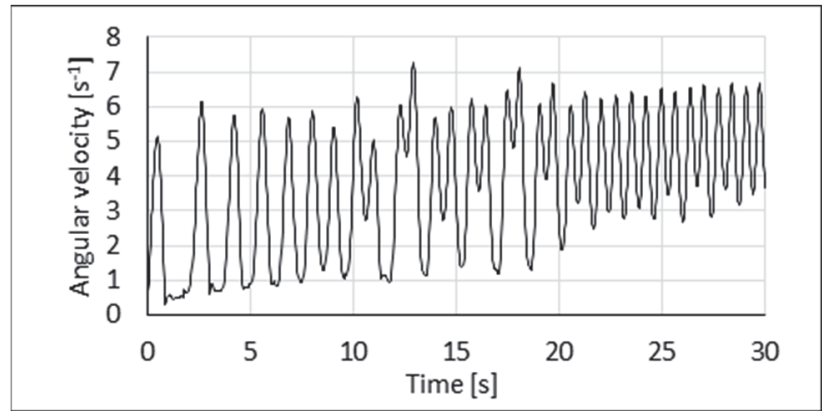

Figure 11 Time distribution of angular velocity in numerical simulation

Under some conditions of oscillation, however, an almost uniform rotation of the axle weights was obtained. Knowing that this cannot be achieved often in practice, numerical simulations for ideal cases were performed, mainly because the best possible operating conditions and the highest possible generation of electricity want to be determined.

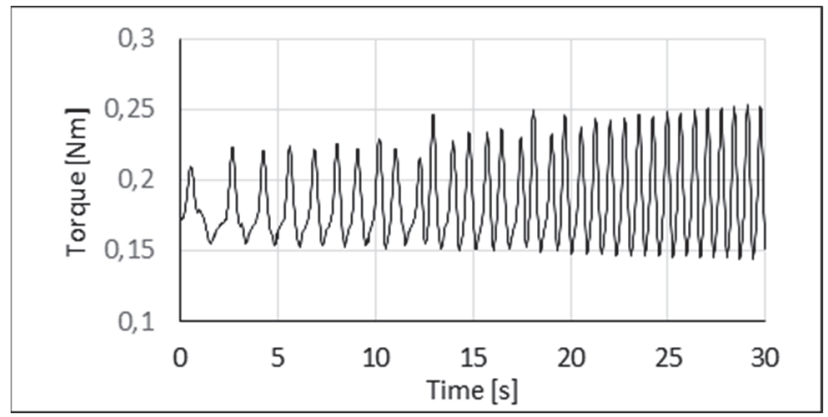

Figure 12 Time distribution of torque in numerical simulation

In fact, motion will produce very different external amplitudes and frequencies. In order to achieve the best possible results of electricity generation, the first situation described above should be avoided. This could be achieved with different generator by changing the induced resistance, which should depend on the external oscillation conditions.

Some of the input parameters in numerical simulations simply had to be defined based on some known assumptions. To determine some of numerical parameters more accurately, measurements on a model were performed. For the measurements an industrial robot was used (Fig. 13). To obtain as close as possible operating parameters the detailed measurement for certain position of the weight was done.

Laboratory testing was performed using an $\mathrm{ABB}$ industrial robot [12]. A computer program for controlling the robot was created, where during the measurements the change of the maximum oscillation angle and speed of oscillation can be done. It was also possible to vary the distance of the eccentric weights from the axis of rotation.

In this case, measurements were made from $0,07 \mathrm{~m}$ to $0,15 \mathrm{~m}$. For individual values of weight distances and maximum pendulum angles, the height difference, which is a major parameter in energy measurements, can be calculated.
Comparing the input (potential energy) without considering the robot's work, output power was obtained that exceeds the theoretical value by several percents with all combinations of parameters. This is, of course, due to the fact that the calculations did not take into account the energy used by the robot during this operation.

As a result of the measurements, electrical current and voltage on the DC generator were obtained. At the same time, the speed of rotation was also measured so that the torque on the shaft could be determined.

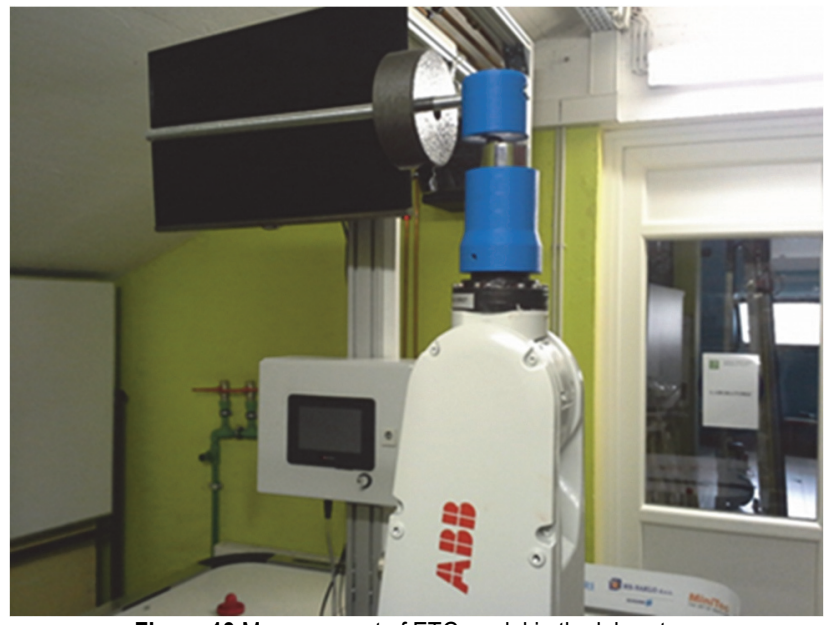

Figure 13 Measurement of ETG model in the laboratory

The power output was calculated from the results of the measurements of voltage and current measured in the laboratory. During the eccentric operation of the robot, the measured values varied greatly over time, so it is not possible to speak about constant power. Therefore, it was focused on maximum values, which in some cases are repeated periodically between measurements.

A similar geometry was also prepared for numerical simulation. The input parameters in the numerical simulation are partially different from the parameters in the measurements, but conditions of analyses were tried to be achieved as similar as possible for both types of analyses. The results of the numerical analyses gave very similar results to those obtained in the laboratory measurements.

In the numerical simulations it was possible to achieve similarity in geometric parameters regarding the shape and position of the weights. The similarity of the materials and thus the weight of the weights was also satisfied. The similarity conditions in the excitation function and in the resistance coefficient, which was absolutely necessary in the numerical simulations could not be completely satisfied.

In the measurements, the resistance is related to the electromagnetic induction in the generator.

The difference between the measurements and the numerical calculations for the different weight positions was between $5 \%$ and $15 \%$, which is a good result for this purpose.

This will allow us to numerically predict the characteristics of the generator at different oscillation modes for different generator geometries, different weights and height differences. 


\section{CONCLUSIONS}

Given the fact that the energy of oscillation is present in various physical processes in nature, it makes sense to think about the exploitation of this energy and the conversion of mechanical energy into electrical energy.

In this paper, two different models that exploit the same energy potential have been presented.

The basic assumption in the presented research is the fact that the oscillation energy is available and is therefore not considered as an input parameter.

Taken into account was also the fact that in a driven harmonic oscillation, where in a pendulum having a known natural frequency and is excited by an external source, resonance can occur. In this case the total oscillation energy, which is initially theoretically determined by the initial potential energy, increases considerably.

In the first phase, the various harmonic motions caused mainly by the force of gravity were analysed theoretically and numerically. It was found out that such a general 3D motion is usually very complex, so we quickly switched from theoretical to numerical investigation.

Since it is necessary to define a large number of physical parameters that affect motion in numerical simulation, parallel measurements using an industrial robot were performed. Based on the measurement results, the numerical results of the model were validated. Further on, it will be possible to numerically analyse different sizes of prototypes in the future, which will be optimized to achieve better energy conversion.

In the continuation of the study of mechanical oscillation, we will also focus on electronics in the future, which will enable the most optimal conversion of mechanical energy into electrical energy using different sensors of motion and continuously monitoring the intensity of electromagnetic induction.

Development has also gone in the direction of the design of the electric generator itself with optimal winding of the coils and the moving magnet, where it is necessary to ensure the least possible friction and total resistance to movement, which is caused mainly by uneven oscillation.

Through measurements as well as numerical analyses, we came to the conclusion that the theoretical power of the electricity generators presented above mainly depends on the initial available potential energy. This depends on the mass of the weights in the case of the ETG generator or on the mass of the moving magnet inside the torus in the case of the TTG generator and the maximum height difference in oscillation.

To achieve the maximum instantaneous power, the data on the potential energy is sufficient, but for the amount of electricity produced the frequency of oscillations or, better said, the time course of the frequency is also very important. Since both characteristics, frequency and amplitude are important in the case of excited oscillation, it is necessary to define the connections between mass, frequency and amplitude for the most optimal energy conversion.

In the continuation of the research, we will also focus on these challenges.

\section{Nomenclature}

$F \quad$ force

$\mathrm{N}$

$F_{\text {a }} \quad$ active force

$F_{\mathrm{b}} \quad$ bonding force

$\mathrm{N}$

$m$ mass

$P \quad$ power

$r$ radius vector

$t$ time

$W \quad$ energy

$\beta \quad$ damping coefficient

$\omega_{\mathrm{cs}} \quad$ angular frequency (coordinate system)

$\omega_{\mathrm{g}} \quad$ angular frequency (magnet/weight)

\section{Acknowledgements}

Research presented in this paper has been conducted as a part of the project OP20.03534 partially financed by the Ministry of Education, Science and Sport, Republic of Slovenia.

\section{REFERENCES}

[1] Falnes, J., (2002). Optimum Control of Oscillation of WaveEnergy Converters. International Journal of Offshore and Polar Engineering, 12(02).

[2] De la Villa J. A., Garcia-Santana, A., \& Montoya-Andrade, D. E. (2014). Maximizing output power of linear generators for wave energy conversion. International Transactions on Electrical Energy Systems. https://doi.org/10.1002/etep.1747

[3] Bertram, D. V. (2020). A systematic approach for selecting suitable wave energy converters for potential wave energy farm sites. Renewable and Sustainable Energy Reviews, 132. https://doi.org/10.1016/j.rser.2020.110011

[4] Josefsson, J. (2015). Numerical Study of Resonant Wave Energy Converters in Selected Sites, Strategic use and Optimal Tuning of the Mechanism for Phase Control. Master Thesis, Royal Institute of Technology.

[5] Tseng, Z. S. (2018). Mechanical vibrations. Department of Mathematics, class/Math251, Penn State.

[6] Schmitz, T. L. \& Smith, K. S. (2012). Mechanical Vibrations Modeling and Measurement. Springer.

[7] Baraff, D. (1997). An Introduction to Physically Based Modelling: Rigid Body Simulation I - Unconstrained Rigid Body Dynamics. Robotics Institute, Carnegie Mellon University.

[8] Smolar, N. \& Virtič, P. (2019). Design investigation of electromechanical generator for energy harvesting. International conference on advances in energy systems and environmental engineering (ASEE19), 116. https://doi.org/10.1051/e3sconf/201911600079

[9] Pirisi, A., Mussetta, M., Grimaccia, F., Caputo, D., Gruosso, G., \& Zich, R. E. (2012). An Innovative Device for Traffic Energy Harvesting. Proceedings of $6^{\text {th }}$ IET PEMD. https://doi.org/10.1049/cp.2012.0337

[10] Asama, J., Burkhardt, M. R., Davoodi, F., \& Burdick, J. W. (2015). Investigation of energy harvesting circuit using a capacitor-sourced buck converter for a tubular linear generator of a moball: A spherical wind-driven exploration robot. IEEE Energy Conversion Congress and Exposition. https://doi.org/10.1109/ECCE.2015.7310104

[11] ANSYS Mechanical Tutorials, (2015). ANSYS, Inc. Southpointe 2600 ANSYS Drive, Canonsburg, PA 15317.

[12] Robotics Product specification IRB 1200, (2018). ABB. 


\section{Contact information:}

\section{Andrej LIPEJ}

(Corresponding author)

University of Novo mesto, Faculty of Mechanical Engineering,

Na Loko 2, 8000 Novo mesto, Slovenia

E-mail: andrej.lipej@fs-unm.si

\section{Matej ŠTEFANIČ}

University of Novo mesto, Faculty of Mechanical Engineering,

Na Loko 2, 8000 Novo mesto, Slovenia

E-mail: matej.stefanic@fs-unm.si

\section{Simon MUHIČ}

University of Novo mesto, Faculty of Mechanical Engineering,

Na Loko 2, 8000 Novo mesto, Slovenia

E-mail: simon.muhic@fs-unm.si

\section{Primož KUNAVER}

Primum d.o.o.,

Mesesnelova ulica 8, 1210 Ljubljana, Slovenia

E-mail: primoz.kunaver@primum.si 